\title{
Is evolution of domestication driven by tameness? A selective review with focus on chickens
}

Beatrix Agnvall, J ohan Bélteky, Rebecca Katajamaa and Per J ensen

The self-archived postprint version of this journal article is available at Linköping University Institutional Repository (DiVA):

http:/ / urn.kb.se/ resolve?urn=urn:nbn:se:liu:diva-150238

N.B.: When citing this work, cite the original publication.

Agnvall, B., Bélteky, J ., Katajamaa, R., J ensen, P., (2018), Is evolution of domestication driven by tameness? A selective review with focus on chickens, Applied Animal Behaviour Science, 205, 227233. https:// doi.org/ 10.1016/j.applanim.2017.09.006

Original publication available at:

https:/ / doi.org/ 10.1016/j.applanim.2017.09.006

Copyright: ELSEVIER SCIENCE BV

Publisher URL Missing

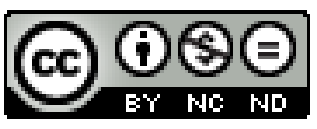




\title{
Is evolution of domestication driven by tameness? A selective review with focus on chickens
}

\author{
Agnvall, Beatrix; Bélteky, Johan; Katajamaa, Rebecca; and Jensen, Per* \\ IFM Biology, AVIAN Behaviour Genomics and Physiology Group \\ Linköping University \\ 58183 Linköping \\ Sweden
}

*Communicating author: per.jensen@liu.se 


\begin{abstract}
Domestication of animals offers unique possibilities to study evolutionary changes caused by similar selection pressures across a range of species. Animals from separate genera tend to develop a suite of phenotypic alterations referred to as "the domesticated phenotype”. This involves changes in appearance, including loss of pigmentation, and alterations in body size and proportions. Furthermore, effects on reproduction and behaviour are typical. It is hypothesized that this recurring phenotype may be secondary effects of the increased tameness that is an inevitable first step in the domestication of any species. We first provide a general overview of observations and experiments from different species and then review in more detail a project attempting to recreate the initial domestication of chickens. Starting from an outbred population of Red Junglefowl, ancestors of all modern chickens, divergent lines were selected based on scores in a standardized fear-of-human test applied to all birds at 12 weeks of age. Up to the eighth selected generation, observations have been made on correlated effects of this selection on various phenotypes. The fear score had a significant heritability and was genetically correlated to several other behavioural traits. Furthermore, low-fear birds were larger at hatch, grew faster, laid larger eggs, had a modified metabolism and increased feed efficiency, had modified social behaviour and reduced brain size. Selection affected gene expression and DNAmethylation in the brains, but the genetic and epigenetic effects were not specifically associated with stress pathways. Further research should be focused on unraveling the genetic and epigenetic mechanisms underlying the correlated side-effects of reduced fear of humans.
\end{abstract}

Keywords: Genetics, epigenetics, domestication, Red Junglefowl

\title{
1. Introduction
}


When Charles Darwin published what was later called the theory of evolution, he devoted the first chapter of his book to domestication. The reason was of course simple: at that time (1859), no proper theory of inheritance and genetics was known, and the fact that species can gradually change due to selection and develop into new varieties was not self-evident to his readers. But domestication served as an important proof-of-concept. If human-imposed selection can result in the rich variety of forms seen, for example, in domesticated pigeons, dogs and garden plants, why could natural selection not achieve the same effects on natural populations?

Hence, domestication as a field of study has a rich and important tradition in biology, and to this day it can serve as a model to explore evolutionary mechanisms and functions. Animal domestication has been defined as the modifications in a population caused by human selection (Price, 2002). It is a genetic process that ranges over generations, and can therefore be considered a special case of evolution where humans drive the process. But the selection imposed by people is only one factor in domestication. Natural selection continues to act on domestic populations, and sexual selection can also have a very high impact (Jensen and Wright, 2013). Furthermore, genetic drift, as well as correlated genetic responses can exert large effects.

Understanding evolution is an important reason for studying domestication, but there are other lessons to be learnt as well. For example, modern selection of farm animals have on average more than doubled their production capacities, while at the same time a range of health problems have become wide-spread as unintended side-effects (Rauw and Kanis, 1998). By examining the mechanisms in domestication we may gain important knowledge necessary to develop breeding programs that do not induce poor animal welfare as side effects.

Domestic animals from widely different species tend to develop a suite of similar phenotypic changes, often referred to as the domesticated phenotype (Jensen, 2014; Price, 2002). This includes, for example, changes in size, pigmentation, body proportions, reproduction and social behaviour. Similar developments happens in species that have been domesticated at different historical time periods and in different parts of the world. For example, the dog was the first domesticated species, 
originating in south-east Asia some 15000 years ago (Ding et al., 2011), and the rabbit is a late addition domesticated about 1400 years ago in southern Europe (Carneiro et al., 2014). In spite of the large differences in time and geographical origin, in both species we find a radiant development of breeds with changes in behaviour, size, pigmentation, as well as skull and ear shapes.

All domestication events show individual differences and peculiarities, but they also share important aspects. Whereas it was long believed that humans have been actively choosing and enforcing domestication on species that they preferred, archeological and genetic data have shown that a period of "proto-domestication" or "predomestication cultivation” preceded actual domestication for most domesticated species (Larson et al., 2014). This means that there has been extensive co-evolution between man and animal, and shared traits in different domesticates could be a result of similar natural selection pressures occurring even before humans applied active selection for preferred traits.

Domestication therefore offers a powerful model for studies of evolution. One may view the process as a gigantic biological experiment, where humans have imposed similar selection pressures on replicated populations of different species at different time periods. With the modern biological tool-box, we can now harvest the results of this unintentional experiment (Andersson and Georges, 2004).

\section{Tameness as a driver?}

Considering the possible common aspects of animal domestication, one trait has been recurrently suggested to be of main importance. Successful domestication of any species must have relied on the animals showing drastically reduced fear of humans. Individuals showing excessive fear and stress reactions in the proximity of humans would have been unlikely to reproduce and thrive in captivity. The Russian geneticist Belyaev expressed it this way: "What do I mean by domesticated behavior? The main criterion here is the ability of animals to have direct contact with man, not to be afraid of man, to obey him, and to reproduce under the conditions created by him (...). It is 
obvious that selection for behavior has been unconsciously carried out by man since the earliest stages of animal domestication.” (Belyaev, 1979).

Could tameness in fact drive the development of other aspects of the domesticated phenotype? Based on this assumption, Belyaev, together with his colleague Lyudmila Trut, selected farmed silver foxes for reduced fear over many generations and measured the occurrence of correlated traits in the selected population (Trut et al., 2009). They found a rapid increase of domesticated phenotypes in the tame animals. For example, the frequency of de-pigmented animals increased, as well as foxes with changes in skull shape, body proportions, and ontogenetic development.

Similar experiments have since been carried out on a few other, unrelated species. For example, the Russian group also selected wild-caught rats for high or low degree of defensive aggression towards humans (Albert et al., 2008; 2012), and Danish scientists have performed similar experiments on farmed mink (Malmkvist and Hansen, 2001). In general, increased tameness appears associated with a range of correlated phenotypic effects.

It therefore remains a plausible possibility that tameness is the driving factor underlying the development of domesticated phenotypes in widely separate species. Belyaev speculated that this could be caused by various hormones that are affected by reduced fear of humans, and simultaneously regulate gene function and developmental patterns (Belyaev, 1979).

\section{Possible genetic mechanisms in domestication}

A number of genetic mechanisms could tentatively cause the changes associated with domestication. The first and most straightforward would be that humans independently in different species selected novel mutations occurring randomly in the tame populations. This would mean that similar types of mutations would have to occur in all species, something Belyaev considered highly unlikely (Belyaev, 1979). He suggested that rather a few central genes might be responsible for a cascade of effects leading to the domesticated phenotype. However, independent and repeated 
selection of random mutations have been suggested to underlie pigment changes in domesticated pigs (Rubin et al., 2012).

Effects of few genes on many phenotypes could be caused by other mechanisms as well: pleiotropy, epistasis, linkage and epigenetics would all be possible (Fig 1). Pleiotropy is the term used when variation in one single gene causes changes in several unrelated phenotypes. For example, a mutation in the gene PMEL17 causes loss of pigmentation in chickens, and at the same time reduces the risk of being exposed to feather-pecking (Keeling et al., 2004). Possibly, genes related to tameness could exert pleiotropic effects on domestication phenotypes in a similar manner.

Linkage refers to when genes situated physically close on a chromosome will be inherited as a unit, causing groups of genes to act as an entity. For example, a small region on chicken chromosome 1 affects traits ranging from growth to reproduction and fear related behaviour, and would be a strong candidate for possible domestication effects in this species (Schütz et al., 2004).

Epistasis is the term that describes when a gene modifies the effect of one or several other, distantly situated genes. For example, early growth in chickens is affected by several gene pairs acting in an epistatic manner (Carlborg, 2003). Here, selection on a mutation in one gene could cause effects on phenotypes controlled by another, epistatically associated with the selected one.

Last, but not least, epigenetic modifications could be central drivers of domestication effects. This refers to heritable modifications of the DNA that affects gene expression without affecting the DNA-sequence (Jensen, 2015). This is a relatively novel field that may in fact have wide ramifications in evolutionary biology, including domestication. Epigenetic modifications may happen very fast and in direct response to environmental challenges, thus potentially explaining the rapid responses observed to selection for tameness. One study found large epigenetic differences in the brains of domesticated chickens compared to their ancestors, the Red Junglefowl, supporting the suggestion that epigenetics may play a large role in evolutionary radiation (Nätt et al., 2012). 
Some studies have observed overlapping genetic factors in different species, suggesting a common genetic mechanism for domestication, whereas others have failed to find the same. For example, one study showed that a chromosomal region associated with increased tameness in Belyaev's foxes is orthologous to a domestication-related region in dogs, implying similar causal grounds in these two species (Kukekova et al., 2010). However, another study failed to find more than occasional similarities in brain gene expression differences between domesticated and wild specimens across a range of species (Albert et al., 2012). Hence, there is a need for further studies to elucidate the underlying genetic mechanisms associated with the development of the domesticated phenotype.

\section{Chickens - important domesticates}

Whereas much can be learnt from the previously mentioned studies of foxes, rats and mink, they all suffer from the same limitation: the species are all historically late domesticates and therefore represent novel domestication attempts. We decided to try another route, namely to replicate domestication under controlled conditions in a species that was domesticated early in history, representing the "classical domesticates”. The natural choice in this respect was the chicken.

Chickens are the most numerous food-producing animals on the planet, exceeding 50 billion individuals (Nicol, 2015). They are also in the center of important animal welfare discussions, since breeding as well as husbandry is by far more intense in this species compared to any other domesticated animal.

All modern chickens originate from the Red Junglefowl, still living wild in India and South-East Asia, and domestication started about 8000 years ago (Tixier-Boichard et al., 2011). Today, there is a great variety of breeds and types, although food producing birds (and hence the majority of individuals in the world) exist in only few varieties (Nicol, 2015). 
In nature, Red Junglefowl live in small harem groups where several males and females together occupy small territories. They are omnivorous, ground-living birds that spend the nights perching in trees and produce one to two litters of chicks per year (Nicol, 2015). During domestication, many fundamental aspects of the natural behaviour have been retained, but much has also been modified in line with the domesticated phenotype described earlier. For example, domesticated chickens are less active and explorative, and show a higher degree of social tolerance to conspecifics than Red Junglefowl (Lindqvist and Jensen, 2009; Schütz and Forkman, 2001).

In spite of being inherently fearful towards humans, Red Junglefowl are possible to keep in captivity under conditions similar to those for domestic chickens. Hence, comparative and experimental studies on this species are feasible, making it an excellent model for a re-domestication experiment.

\section{Re-domesticating chickens}

The aim of our re-domestication project was to select Red Junglefowl based only on their fearfulness towards humans, while rearing them under identical conditions, and measure how traits related to the domesticated phenotype are affected as correlated responses over generations. Furthermore, we collected tissue samples to unravel genetic and epigenetic mechanisms underlying the effects we would observe.

To obtain an outbred and unbiased initial population, resembling the wild ancestor of domesticated chickens, we started from two different captive populations obtained from different zoos. These were reciprocally cross-bred for two generations, thereby producing the initial parental generation (P0), consisting of about 100 birds, on which our selection program started (Agnvall et al., 2012).

For all birds in the project, starting with the P0 and continuing in every generation, their behaviour was recorded in a fear-of-human test when they were 12 weeks old. In this test, each bird was individually placed in an arena, and during three minutes a human moved through the arena according to a standardized pattern, and in the end 
attempted to touch the bird. The behaviour was recorded throughout the test and used to form a fear score for each individual. Detailed information can be found in Agnvall et al. (2012).

In the first generation, we selected about $25 \%$ with the highest fear scores, and the 25 \% with lowest to form the High Fear $(\mathrm{H})$ and Low Fear $(\mathrm{L})$ selection lines. The remaining birds were randomly mated to form an Unselected (U) control line. In subsequent generations, we selected the most fearful $\mathrm{H}$-birds and the least fearful Lbirds as parents, and continued to randomly breed U-birds. More details can be found in Agnvall et al. (2012).

All chickens were weighed at hatch and throughout life, and a series of behaviour tests were carried out at standardized ages. In addition to that, some tests and experiments were carried out in different generations as new ideas and hypotheses emerged during the course of the project. When the birds were culled, we collected various tissue samples for later analysis of gene expression and morphometric studies. A summary of the most important effects observed in the selected populations is found in Table 1, and these are further dealt with in some detail below.

\section{Heritabilities and genetic correlations of tameness}

It is not totally obvious that tameness should have a genetic basis, not least in chickens. Newly hatched chicks imprint on large objects, for example, humans, and any observed difference in tameness could potentially be a result of differences in early experiences. In order to establish the genetic contribution, we mimised differences in early experiences by standardizing routines at hatching and treating all birds equally.

We calculated heritabilities and other genetic estimates in the second selected generation (S2). There was a significant effect of selection group on the fear-ofhuman trait, and the heritability $\left(\mathrm{h}^{2}\right)$ was estimated to 0.17 (Agnvall et al., 2012). This shows that a significant part of the variation in tameness could be attributed to genetic variation. 
Furthermore, there were several significant genetic correlations $\left(\mathrm{r}_{\mathrm{s}}\right.$ ) between the fear score and other behavioural traits recorded, with absolute values mostly ranging between $0.1<\mathrm{r}_{\mathrm{s}}<0.6$ (Agnvall et al., 2012). For example, activity and reactions in a social reinstatement test had significant genetic correlations with fear score. Hence, it could be predicted that social behaviour would change as a result of selection.

\section{Growth and metabolism}

As seen above, size changes relative to the ancestors are common in domestic animals, and an integral part of the domesticated phenotype. Could this be related to increased tameness?

Already in S2 there was a significant effect of selection on hatch weight, where Lbirds were heavier (Agnvall et al., 2012). This was further investigated in the fourth selected generation (S4). Here, we found that L-birds laid larger eggs and had larger offspring than H-birds (Agnvall et al., 2014). This would also be in line with what could be expected from the domesticated phenotype, since modifications in reproductive physiology is an integral aspect (Price, 2002). Furthermore, L-birds were significantly larger at 200 days of age.

We considered the possibility that the growth differences could reflect variation in metabolism, possibly related to reduced stress in the L-birds. Therefore, we measured basic metabolic rates (BMR) using open-flow respirometry in five-week old birds from the S5 generation (Agnvall et al., 2015). L-birds of both sexes had significantly higher BMR. In addition, we assessed feed intake and growth in adult birds from the same generation, and found that females from the L-birds had a higher feed efficiency (g growth per ingested g feed; G:F).

The L-birds in the same generation were also bolder in a Novel Object test, indicating that the assumption of lower stress levels in tamer birds could be a possible explanation for the growth differences. The growth differences were also accompanied by higher blood serotonin levels in the L-males. However, in neither sex 
were there any effects of selection for tameness on corticosterone reactions to a brief physical restraint. Hence, it is still not clear how the reduced fear of humans generalizes to reduced stress in general.

\section{Social behaviour}

The domesticated phenotype is associated with changes in social behaviour that may facilitate living in large groups under confined conditions (Price, 2002). As mentioned above, we found genetic correlations between fear of humans and social reinstatement behaviour already in the second selected generation, so we decided to carry out more detailed experiments on selection side effects on social behaviour.

We compared the general social behaviour in groups of three same-sex birds from the selection lines in the fifth generation (S5) and did not find any differences (Agnvall et al., 2014). Hence, the general level of social interactions was not affected by the selection. However, when the groups were instead composed of one bird from each

selection line (L, H, U), the low fear birds performed more feeding, more aggression, and were more dominant.

We suspected that this effect could be a result of L-birds growing faster, and hence have an increased feeding motivation. We therefore replicated the experiment, again with groups of three same-sex birds, one from each selection line, in tests not involving competition over feed (Agnvall et al., 2014). After an initial deprivation period, the birds were offered limited access to either a dust bath or water. In both cases, the L-birds showed more aggressive behaviour and were dominant in the competition over the limited resource as also seen in the previous test. Hence, we concluded that tameness is related to increased social dominance, perhaps again as a result of a generally reduced level of fear and stress in the test situation.

\section{Size of brain and other organs}

The most obvious effect of domestication is a change in morphology. Allometric changes in organ sizes, including the brain, have been observed in all domestic 
species (Diamond, 2002; Price, 2002; Trut et al., 2009). As mentioned above, selection for tameness in our Red Junglefowl population caused a rapid correlated effect on body size and growth, where tame birds hatched larger and grew faster.

We measured the sizes of the brains and other organs in the parental P0 and the fifth selected generation (S5) (Agnvall et al, in press). Absolute as well as relative total brain sizes (proportion of body weight) were on average smaller in the L-birds. This is in accordance with general observations that domesticated variants usually have reduced brain size compared to their wild ancestors (Kruska, 2005; Price, 2002).

Although the brain as an entity was reduced in size by selection for increased tameness, this did not pertain to all brain parts equally. We divided the brains into four different parts and weighed each of them separately. This showed that the relative weight of cerebellum (proportion of total brain weight) was significantly larger in the L-birds, in spite of them having overall smaller brains (Agnvall et al, in press). Hence, it appears that the relative size of this region does not respond to the selection like the rest of brain. The specific function of the cerebellum is not understood in all details, and recent research suggests that it may be more involved in social behaviour than what has previously been known (Barton, 2012). The varying selection effects on different brain regions is consistent with recent studies showing that the sizes of different parts of the brain are under genetic control of different loci (Henriksen et al., 2016).

Other organs were also found to respond in a correlated fashion to the selection process. The relative weights (proportion of body weight) of the heart, liver, testes and spleen were all significantly lower in the L-birds. This could perhaps be a result of resource reallocation caused by a generally lower stress level in the L-birds, or of the fact that the increased body weight mainly comes from increased sizes of muscles, fat and bones.

It was also observed that birds from the $\mathrm{H}$ line had a poorer feather condition in the S3-generation, indicating that they were more exposed to feather-pecking (Agnvall et al., 2014). Since the birds were all housed in mixed-group pens, with birds from all 
three selection lines together, this indicates that something in the behaviour or appearance of individual birds increase the risk of being exposed to feather pecking. For example, victims of feather pecking have been found to be more asymmetrical than non-pecked birds (Tahamtani et al, 2017). This is consistent with previous findings, that chickens with a mutation in PMEL17, causing loss of pigmentation, are less exposed to feather-pecking than wildtype-coloured birds (Keeling et al., 2004). Loss of pigmentation is a hallmark of the domesticated phenotype, and interestingly, in the $8^{\text {th }}$ selected generation a few birds in the low fear selection line have been detected with untypical white patches, something so far not observed in the $\mathrm{H}$ line or in earlier generations of L line birds (Fig 2) (Katajamaa, unpublished data). This observation is preliminary and has only been noted in one or two birds, so it remains to be seen whether this phenotype will increase among the L-birds in subsequent generations.

\section{Gene expression and epigenetics}

Previously, comprehensive analyses of genetic sweeps (genomic regions associated with selection) have identified a large number of genomic regions that have been under selection during chicken domestication (Rubin et al., 2010). These and other studies strongly indicate that the main selection responses affect regulatory regions (for example, promoters and enhancers) rather than protein-coding sequences. Hence, we would expect rapid selection responses in gene expression patterns.

We analysed gene expression differences in the hypothalamus/thalamus and the cerebral hemispheres of the P0 and S5 generations, using microarrays to achieve a genome-wide assessment (Beltéky et al., 2017; 2016). The results showed a limited overlap between the brain regions, again indicating that different parts of the brain respond differently to the selection. Functional analysis of the annotated genes showed over-representation of pathways associated with behaviour in the cerebral hemispheres, and processes such as reproduction and immunology in the hypothalamus/thalamus. This shows that selection for tameness caused genetic changes in correlated responses and that affected processes were specific for each brain region. 
A possible mechanism for the rapid responses in phenotype and gene expression could be epigenetic modifications of the genome. For example, DNA-methylation can respond to environmental challenges (such as stress) and can be transgenerationally stable (Guerrero-Bosagna and Skinner, 2014; Jablonka, 2013). In the same brain samples as those used for gene expression, we also isolated mRNA and used methylated DNA-immunoprecipitation (meDIP) and RNA-sequencing to analyse differentially methylated genomic regions between the selection lines (Bélteky et al, manuscript under revision). Although the functional annotation of genes in the differentially methylated regions showed over-representation of metabolic and signaling pathways (indicating effects on similar processes as shown by the gene expression results), there was no overlap between differentially methylated and differentially expressed genes. This indicates that selection has affected genome regions situated far from the target genes, and has modified gene expression mainly through trans-effects.

\section{General discussion}

The studies of farmed foxes by Belyaev and colleagues (Trut et al., 2009) advanced our understanding of domestication in an unprecedented way. However, foxes have only been farmed for just over 100 years, and have therefore not been domesticated like the classic species from early agriculture and before that. Our experiments on chickens are an attempt to replicate the process in a species that has gone through successful classical domestication, and where present-day chickens world-wide show us what happens after thousands of years of selection in this species.

Although limited so far to a few generations, the results are quite interesting. As a result of selection only for reduced fear of humans Red Junglefowl developed a range of features that are consistent with the domesticated phenotype: increased size, growth and reproduction, changes in metabolism and feed efficiency, modified social behaviour, reduced brain size, and possibly loss of pigmentation. These findings together indicate that tameness may have been the driving force behind the complex of traits referred to as the domesticated phenotype. This is consistent with studies of farmed foxes (Trut et al., 2009) and also with the few existing similar studies of 
other species, mainly rats and mink (Albert et al., 2008; Malmkvist and Hansen, 2001).

The mechanisms underlying this remain elusive at this point. It has recently been suggested that the domesticated phenotype could be caused by a general embryological effect acting on the neural crest cells (Sánchez-Villagra et al., 2016). Even if this would be the case, the underlying genetic mechanisms need to be identified. In our analysis of gene expression in different parts of the brain, we have so far found little evidence that any particular gene or gene complex could be causing the wide-ranging effects in the selected Red Junglefowl. One possible hypothesis could be that tameness is related to some central aspect of the stress response, which in turn might affect metabolism and central hormone systems. This is in line with Belyaev’s original suggestions (Belyaev, 1979). However, Kukekova and colleagues found no support for any central genomic region affecting many traits in Belyaev's foxes (Nelson et al., 2016). In fact, different loci were even associated with different parts of the reactions in the fear-of-human test, indicating a complex genetic control. In chickens, we have identified a few candidate genes related to domesticationinduced modifications of stress responses in chickens (Fallahsharoudi et al., 2016), but none of these have turned up in the genetic analysis of the selected Red Junglefowl.

Another possibility is that epigenetic mechanisms are main drivers of the effects observed. For example, DNA-methylation might be affected by stress associated with fear of humans, and individuals able to mount epigenetic responses could in theory be able to adapt more efficiently to captivity. Our results show that few generations of selection was associated with tissue-specific epigenetic modifications, consistent with previous studies showing large epigenetic differences between domestic and ancestral chickens (Nätt et al., 2012). However, to complicate matters, epigenetic variation is partly dependent on genetic variation, so it is possible that selection acts on genotypes with an ability to mount epigenetic responses (Richards, 2006).

Our results suggest that future research needs to focus on two different aspects. Firstly, we need to map the genetic differences between the tame and fearful lines of 
Red Junglefowl, for example, using quantitative trait locus analysis. Secondly, we need to thoroughly analyse the genotype $\mathrm{x}$ epigenotype $\mathrm{x}$ phenotype matrix related to chicken domestication. This involves analysis of how different genotypes are associated with DNA-methylation, and how this in turn relates to the phenotypic variation observed in chickens.

\section{Conclusions}

Animal populations of unrelated species show similar and fast responses to selection for increased tameness. These changes are generally in the direction of the domesticated phenotype, a complex of traits shared by most domesticated species. Red Junglefowl, ancestors of domestic chickens, selected for reduced fear of humans changed accordingly in few generations: tame birds were larger at hatch, grew faster, laid larger eggs, had a modified metabolism and increased feed efficiency, had modified social behaviour and reduced brain size. Selection affected gene expression and DNA-methylation in the brains, but the genetic and epigenetic effects were not specifically associated with stress pathways. Further research is needed to unravel the genetic and epigenetic mechanisms underlying domestication changes in chickens, and in other species.

\section{Acknowledgements}

The research presented in this paper was supported by the Swedish Research Council for Environment, Agricultural Sciences and Spatial Planning (FORMAS), the Swedish Research Council (VR) and the European Research Council (ERC; Advanced Grant 322206 to Per Jensen). 


\section{References}

Agnvall, B., Ali, A., Olby, S., Jensen, P., 2014. Red Junglefowl (Gallus gallus) selected for low fear of humans are larger, more dominant and produce larger offspring. Animal 8, 1498-1505. doi:10.1017/S1751731114001426

Agnvall, B., Jöngren, M., Strandberg, E., Jensen, P., 2012. Heritability and Genetic Correlations of Fear-Related Behaviour in Red Junglefowl-Possible Implications for Early Domestication. PLoS ONE 7, e35162. doi:10.1371/journal.pone.0035162

Agnvall, B., Katajamaa, R., Altimiras, J., Jensen, P., 2015. Is domestication driven by reduced fear of humans? Boldness, metabolism and serotonin levels in divergently selected red junglefowl (Gallus gallus). Biology Letters 11, 20150509. doi:10.1098/rsbl.2015.0509

Agnvall, B., Bélteky, J., Jensen, P., 2017. Brain size is reduced by selection for tameness in Red Junglefowl - correlated effects in vital organs. Scientific Reports 7:3306. DOI:10.1038/s41598-017-03236-4

Albert, F.W., Shchepina, O., Winter, C., Römpler, H., Teupser, D., Palme, R., Ceglarek, U., Kratzsch, J., Sohr, R., Trut, L.N., Thiery, J., Morgenstern, R., Plyusnina, I.Z., Schöneberg, T., Pääbo, S., 2008. Phenotypic differences in behavior, physiology and neurochemistry between rats selected for tameness and for defensive aggression towards humans. Hormones and Behavior 53, 413-421. doi:10.1016/j.yhbeh.2007.11.010

Albert, F.W., Somel, M., Carneiro, M., Aximu-Petri, A., Halbwax, M., Thalmann, O., Blanco-Aguiar, J.A., Plyusnina, I.Z., Trut, L., Villafuerte, R., Ferrand, N., Kaiser, S., Jensen, P., Pääbo, S., 2012. A comparison of brain gene expression levels in domesticated and wild animals. PLoS Genetics 8, e1002962. doi:10.1371/journal.pgen.1002962

Andersson, L., Georges, M., 2004. Domestic-animal genomics: deciphering the genetics of complex traits. Nat Rev Genet 5, 202-212. doi:10.1038/nrg1294

Barton, R.A., 2012. Embodied cognitive evolution and the cerebellum. Phil. Trans. R. Soc. B 367, 2097-2107. doi:10.1098/rstb.2012.0112

Beltéky, J., Agnvall, B., Jensen, P., 2017. Gene expression of behaviorally relevant genes in the cerebral hemisphere changes after selection for tameness in Red Junglefowl. PLoS ONE 12, e0177004. doi:10.1371/journal.pone.0177004

Beltéky, J., Agnvall, B., Johnsson, M., Wright, D., Jensen, P., 2016. Domestication and tameness: brain gene expression in red junglefowl selected for less fear of humans suggests effects on reproduction and immunology. Royal Society Open Science 3, 160033. doi:10.1098/rsos.160033

Beltéky, J., Agnvall, B., Guerrero-Bosagna, C., Höglund, A., Jensen, P., 2017. Epigenetics and early domestication: Hypothalamic DNA-methylation differences in Red Junglefowl divergently selected for reduced fear of humans. Submitted manuscript.

Belyaev, D.K., 1979. Destabilizing selection as a factor in domestication. Journal of Heredity 70, 301-308. doi:10.1093/oxfordjournals.jhered.a109263

Carlborg, 0., 2003. A Global Search Reveals Epistatic Interaction Between QTL for Early Growth in the Chicken. Genome Research 13, 413-421.

doi:10.1101/gr.528003 
Carneiro, M., Rubin, C.-J., Di Palma, Federica, Albert, F.W., Alföldi, J., Barrio, A.M., Pielberg, G., Rafati, N., Sayyab, S., Turner-Maier, J., 2014. Rabbit genome analysis reveals a polygenic basis for phenotypic change during domestication. Science 345, 1074-1079.

Diamond, J., 2002. Evolution, consequences and future of plant and animal domestication. Nature 418, 700-707. doi:10.1038/nature01019

Ding, Z.-L., Oskarsson, M., Ardalan, A., Angleby, H., Dahlgren, L.-G., Tepeli, C., Kirkness, E., Savolainen, P., Zhang, Y.P., 2011. Origins of domestic dog in Southern East Asia is supported by analysis of Y-chromosome DNA. Heredity 108, 507-514. doi:10.1038/hdy.2011.114

Fallahsharoudi, A., de Kock, N., Johnsson, M., Bektic, L., Ubhayasekera, S.J.K.A., Bergquist, J., Wright, D., Jensen, P., 2016. Genetic and Targeted eQTL Mapping Reveals Strong Candidate Genes Modulating the Stress Response During Chicken Domestication. Genes Genoms Genetics G3 7, g3.116.037721-504. doi:10.1534/g3.116.037721

Guerrero-Bosagna, C., Skinner, M.K., 2014. Environmentally induced epigenetic transgenerational inheritance of male infertility. Current Opinion in Genetics \& Development 26, 79-88. doi:10.1016/j.gde.2014.06.005

Henriksen, R., Johnsson, M., Andersson, L., Jensen, P., Wright, D., 2016. The domesticated brain: genetics of brain mass and brain structure in an avian species. Sci Rep 6, 34031. doi:10.1038/srep34031

Jablonka, E., 2013. Epigenetic inheritance and plasticity: The responsive germline. Prog. Biophys. Mol. Biol. 111, 99-107. doi:10.1016/j.pbiomolbio.2012.08.014

Jensen, P., 2014. Behavior Genetics and the Domestication of Animals. Annu. Rev. Anim. Biosci. 2, 85-104. doi:10.1146/annurev-animal-022513-114135

Jensen, P., 2015. Adding "epi-" to behaviour genetics: implications for animal domestication. Journal of Experimental Biology 218, 32-40. doi:10.1242/jeb.106799

Jensen, P., Wright, D., 2013. Behavioral genetics and animal science. In: Grandin, T and Deesing, M.J., Genetics and the behavior of domestic animals, pp 41-80. Amsterdam, Netherlands: Academic Press.

Keeling, L., Andersson, L., Schütz, K.E., Kerje, S., 2004. Chicken genomics: Featherpecking and victim pigmentation. Nature 431, 645-646.

Kruska, D.C.T., 2005. On the evolutionary significance of encephalization in some eutherian mammals: effects of adaptive radiation, domestication, and feralization. Brain Behav Evol 65, 73-108. doi:10.1159/000082979

Kukekova, A.V., Trut, L.N., Chase, K., Kharlamova, A.V., Johnson, J.L., Temnykh, S.V., Oskina, I.N., Gulevich, R.G., Vladimirova, A.V., Klebanov, S., Shepeleva, D.V., Shikhevich, S.G., Acland, G.M., Lark, K.G., 2010. Mapping Loci for Fox Domestication: Deconstruction/Reconstruction of a Behavioral Phenotype. Behav Genet 41, 593-606. doi:10.1007/s10519-010-9418-1

Larson, G., Piperno, D.R., Allaby, R.G., Purugganan, M.D., Andersson, L., ArroyoKalin, M., Barton, L., Climer Vigueira, C., Denham, T., Dobney, K., Doust, A.N., Gepts, P., Gilbert, M.T.P., Gremillion, K.J., Lucas, L., Lukens, L., Marshall, F.B., Olsen, K.M., Pires, J.C., Richerson, P.J., Rubio de Casas, R., Sanjur, O.I., Thomas, M.G., Fuller, D.Q., 2014. Current perspectives and the future of domestication studies. Proceedings of the National Academy of Sciences 111, 6139-6146. doi:10.1073/pnas.1323964111 
Lindqvist, C., Jensen, P., 2009. Domestication and stress effects on contrafreeloading and spatial learning performance in red jungle fowl (Gallus gallus) and White Leghorn layers. Behavioural Processes 81, 80-84. doi:10.1016/j.beproc.2009.02.005

Malmkvist, J., Hansen, S.W., 2001. The Welfare of Farmed Mink (Mustela Vison) in Relation to Behavioural Selection: A Review. Anim Welfare 10, 41-52.

Nätt, D., Rubin, C.-J., Wright, D., Johnsson, M., Beltéky, J., Andersson, L., Jensen, P., 2012. Heritable genome-wide variation of gene expression and promoter methylation between wild and domesticated chickens. BMC Genomics 13, 59. doi:10.1186/1471-2164-13-59

Nelson, R.M., Temnykh, S.V., Johnson, J.L., Kharlamova, A.V., Vladimirova, A.V., Gulevich, R.G., Shepeleva, D.V., Oskina, I.N., Acland, G.M., Rönnegård, L., Trut, L.N., Carlborg, O., Kukekova, A.V., 2016. Genetics of Interactive Behavior in Silver Foxes (Vulpes vulpes). Behav Genet 47, 88-101. doi:10.1007/s10519016-9815-1

Nicol, C.J., 2015. The behavioural biology of chickens. CABI, Wallingford.192 pp

Price, E.O., 2002. Animal Domestication and Behavior. CABI, Wallingford. doi:10.1079/9780851995977.0000

Rauw, W.M., Kanis, E., 1998. ScienceDirect - Livestock Production Science : Undesirable side effects of selection for high production efficiency in farm animals: a review. Livestock Production 56, 15-33.

Richards, E.J., 2006. Inherited epigenetic variation-revisiting soft inheritance. Nat Rev Genet 7, 395-401.

Rubin, C.-J., Zody, M.C., Eriksson, J., Meadows, J.R.S., Sherwood, E., Webster, M.T., Jiang, L., Ingman, M., Sharpe, T., Ka, S., Hallböök, F., Besnier, F., Carlborg, O., Bed'hom, B., Tixier-Boichard, M., Jensen, P., Siegel, P., Lindblad-Toh, K., Andersson, L., 2010. Whole-genome resequencing reveals loci under selection during chicken domestication. Nature 464, 587-591. doi:10.1038/nature08832

Rubin, C.J., Megens, H.J., Barrio, A.M., Maqbool, K., Sayyab, S., Schwochow, D., Wang, C., Carlborg, O., Jern, P., Jorgensen, C.B., Archibald, A.L., Fredholm, M., Groenen, M.A.M., Andersson, L., 2012. Strong signatures of selection in the domestic pig genome. Proceedings of the National Academy of Sciences 109, 19529-19536. doi:10.1073/pnas.1217149109

Sánchez-Villagra, M.R., Geiger, M., Schneider, R.A., 2016. The taming of the neural crest: a developmental perspective on the origins of morphological covariation in domesticated mammals. Royal Society Open Science 3, 160107-541. doi:10.1098/rsos.160107

Schütz, K.E., Forkman, B., 2001. Domestication effects on foraging strategy, social behaviour and different fear responses: a comparison between the red junglefowl (Gallus gallus) and a modern layer strain. Applied Animal Behaviour Science 74, 1-14.

Schütz, K.E., Kerje, S., Jacobsson, L., Forkman, B., Carlborg, O., Andersson, L., Jensen, P., 2004. Major growth QTLs in fowl are related to fearful behavior: possible genetic links between fear responses and production traits in a red junglefowl x white leghorn intercross. Behav Genet 34, 121-130. doi:10.1023/B:BEGE.0000009481.98336.fc 
Tahamtani, F.M., Forkman, B., Hinrichsen, L.K., Riber, A.B., 2017. Both feather peckers and victims are more asymmetrical than control hens. Applied Animal Behaviour Science. doi:10.1016/j.applanim.2017.05.022

Tixier-Boichard, M., Bed'hom, B., Rognon, X., 2011. Chicken domestication: From archeology to genomics. Comptes Rendus Biologies 334, 197-204. doi:10.1016/j.crvi.2010.12.012

Trut, L., Oskina, I., Kharlamova, A., 2009. Animal evolution during domestication: the domesticated fox as a model. Bioessays 31, 349-360.

doi:10.1002/bies.200800070 
Table 1

Summary of the selection responses in phenotypes commonly associated with domestication, observed in the first eight generations of Red Junglefowl selected for reduced fear of humans. All comparisons are in relation to the opposite selection line (high fear of humans).

\begin{tabular}{|c|c|}
\hline Phenotype & Type of change \\
\hline Size & $\begin{array}{l}\text { Increased hatch weight } \\
\text { Increased growth }\end{array}$ \\
\hline Metabolism & Increased basal metabolic rate \\
\hline Feed conversion & Increased growth per g food intake (females) \\
\hline Hormones & Increased blood serotonin (males) \\
\hline Boldness & Reduced latency to approach a Novel Object \\
\hline Social behaviour & Increased social dominance \\
\hline Organ size & $\begin{array}{l}\text { Reduced brain size } \\
\text { Reduced size of heart, liver, testes and spleen }\end{array}$ \\
\hline Plumage & $\begin{array}{l}\text { Increased plumage quality } \\
\text { Possibly loss of pigmentation }\end{array}$ \\
\hline
\end{tabular}


Legend to figures:

Fig. 1

Schematic outline of four possible genetic mechanisms that may cause correlated selection responses in two seemingly unrelated traits. The figure shows a schematic part of a chromosome with two separate genes, G1 and G2. (A) Pleiotropy, in which the genotype of G1 affects the phenotype of both traits simultaneously. (B) Linkage, in which G1 and G2 are linked due to close physical co-localisation, and hence inherited as a unit. (C) Epistasis, in which the effects of G2 on trait 2 depends on the genotype on G1. (D) Epigenetics, in which G1 is epigenetically modified (for example by DNA-methylation) and thereby affects the expression of both G1 and G2.

Fig. 2

Photos showing the loss of pigmentation observed in a bird in generation 8 of the Red Junglefowl selected for reduced fear of humans (A), compared to a normal, wild-type colouration of the same wing area (B). (Photo: R. Katajamaa) 


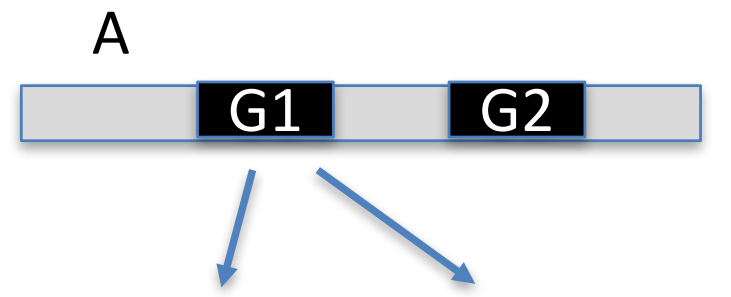

Trait 1 Trait 2
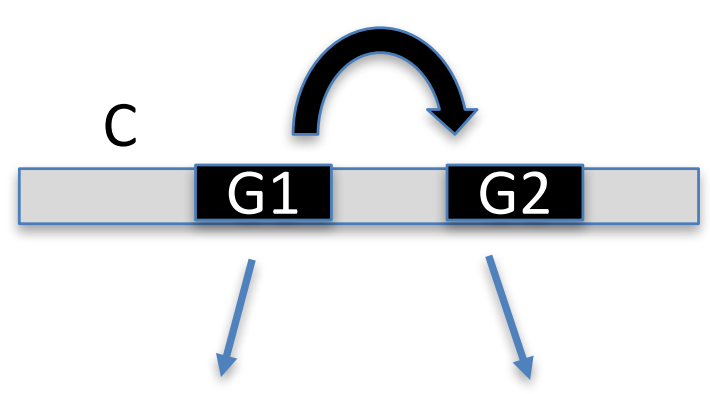

Trait 1 Trait 2

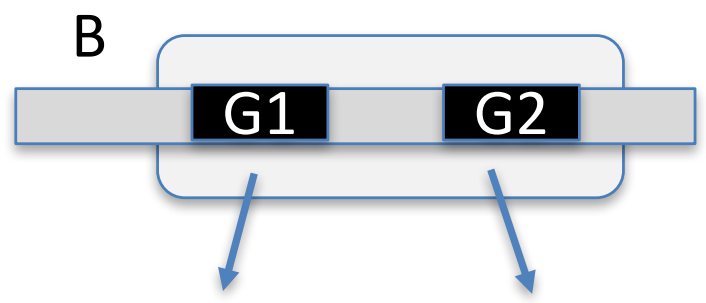

Trait 1 Trait 2

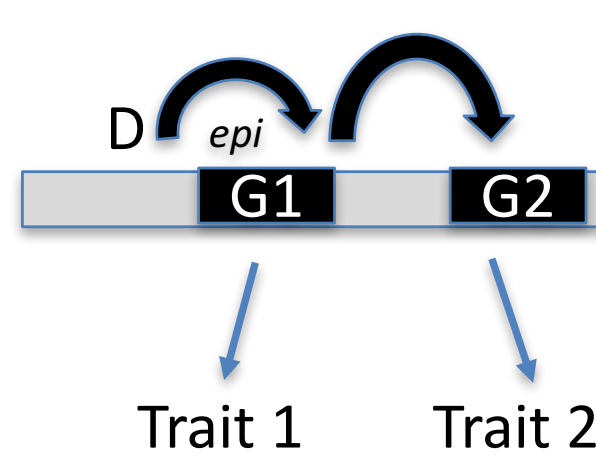

Fig 1 

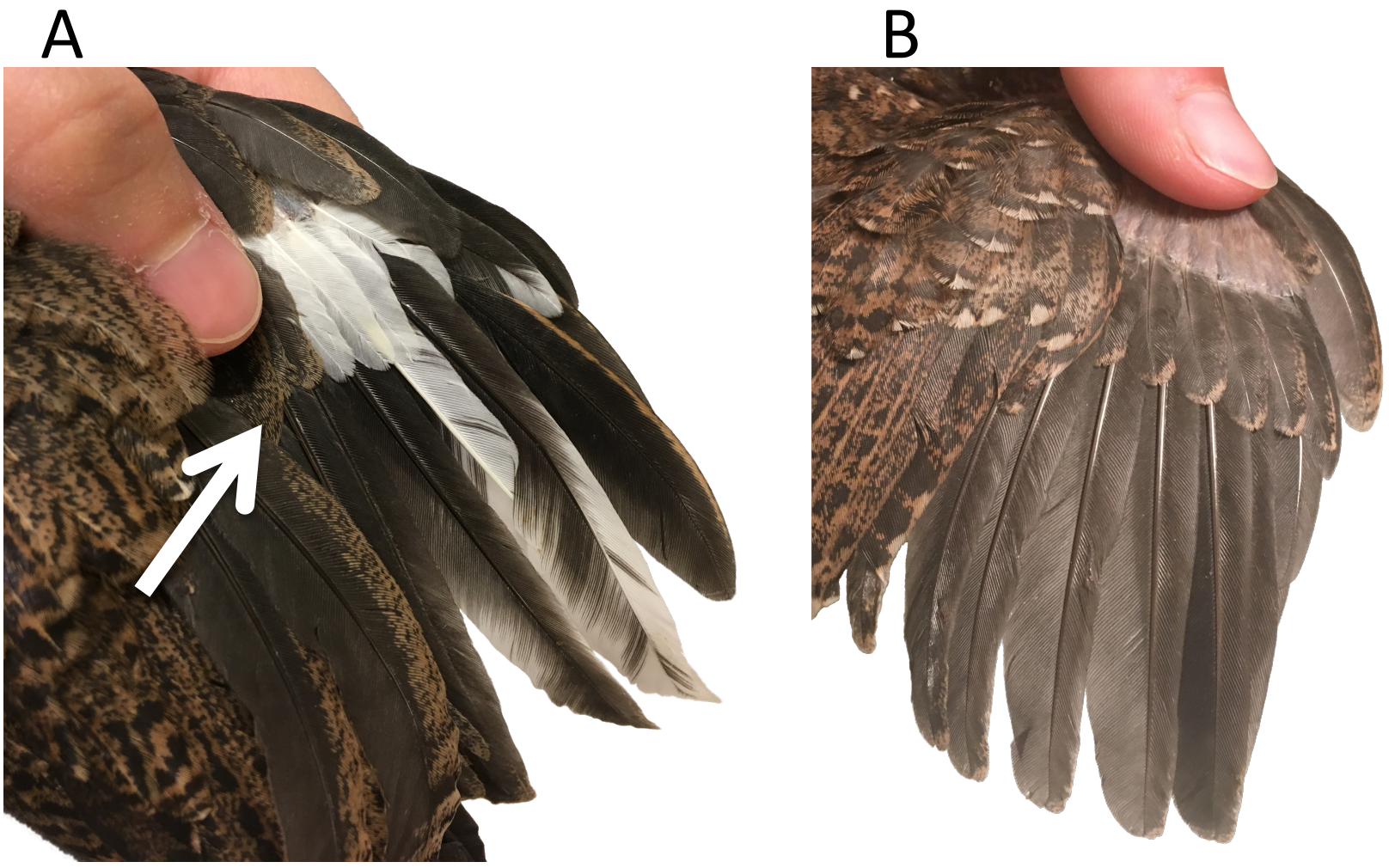

Fig 2 